\title{
EFFECT OF HYDROPHILIC POLYMER IN THREE GREEN ROOF SUBSTRATES ON GROWTH, FLOWER DEVELOPMENT, AND OVERWINTERING OF AGASTACHE RUGOSA (KOREAN MINT) WITHOUT IRRIGATION
}

\author{
XU, H. ${ }^{1}-$ YeUM, K.-J. ${ }^{2}-$ YoON, Y.-H. ${ }^{1}-$ JU, J.-H. ${ }^{3 *}$ \\ ${ }^{1}$ College of Landscape Architecture, Central South University of Forestry and Technology \\ No. 498 Shaoshan South Road, 410004 Changsha, China \\ ${ }^{2}$ Division of Food Bioscience, KonKuk University, 268 Chungwondaero, 27478 Chungju-si, \\ Chungcheongbuk-do, South Korea \\ ${ }^{3}$ Department of Green Convergence Technology, KonKuk University, 268 Chungwondaero, \\ 27478 Chungju-si, Chungcheongbuk-do, South Korea \\ *Corresponding author \\ e-mail: jjhkkc@kku.ac.kr; phone: +82-43-840-3541; fax:+82-43-851-4169
}

(Received 27 ${ }^{\text {th }}$ Apr 2018; accepted 11 $1^{\text {th }}$ Jul 2018)

\begin{abstract}
Some herb plants can be adapted to green roof systems when provided with better growing conditions such as irrigation. The aim of this study was to determine the effects of different amounts of water retentive, hydrophilic polymers used in green roof substrate mixtures, on the growth of Agastache rugosa. Coir dust and perlite were mixed at a ratio of 80 to $20 \%$ (coir dust to perlite, v/v) (termed $\mathrm{C}_{4} \mathrm{P}_{1}$ ), 50 to $50 \%\left(\mathrm{C}_{1} \mathrm{P}_{1}\right)$ or 20 to $80 \%\left(\mathrm{C}_{1} \mathrm{P}_{4}\right)$ at a substrate depth of $20 \mathrm{~cm}$. Hydrophilic polymer was added to the substrate mixtures at 0 (polymer: medium (w/v), dry weight basis) (control), 0.25, 0.5, 1.0 or 2.0 $\mathrm{kg} \cdot \mathrm{m}^{-3}$. Our study indicated that Agastache rugosa can withstand extended dry conditions experienced in green roofs. Substrates $\mathrm{C}_{4} \mathrm{P}_{1}$ and $\mathrm{C}_{1} \mathrm{P}_{1}$ with high coir dust matter are not suitable for Agastache rugosa because of the low growth and poor ornamental quality observed in these plants, especially during the summer rainy season. For the determination of difference in growth, flower development and overwintering of Agastache rugosa among the different substrate conditions, Duncan's multiple range test was conducted. It was found that the addition of $1.0 \mathrm{~kg} \cdot \mathrm{m}^{-3}$ hydrophilic polymer to $\mathrm{C}_{1} \mathrm{P}_{4}$ significantly increased the number of inflorescences and resulted in good growth and ornamental quality during both drought and rainy seasons. Moreover, higher overwintering rate was associated with lower coir dust and hydrophilic polymer content. These results indicated that addition of $1.0 \mathrm{~kg} \cdot \mathrm{m}^{-3}$ hydrophilic polymer to $\mathrm{C}_{1} \mathrm{P}_{4}$ was optimal for the growth of Agastache rugosa in green roofs under dry and rainy seasons.
\end{abstract}

Keywords: rooftop, hydrogel, native herb, water content, urban agriculture

\section{Introduction}

Green roof is an environmentally friendly way to reduce urban heat island, noise and air pollution. At the same time, it can improve storm water management with delayed runoff, water quality, building's microclimate as well as aesthetic value and habitats for wildlife (Santamouris, 2014). Because of their numerous environment benefits, and more green, and open-air amenities without additional land acquisition cost, green roofs has become popular in many cities around the world. However, environmental conditions on the rooftops are often extreme compared to those on the ground, such as high temperature, strong wind, and long-term drought. Extensive green roof with light and thin layer of substrate demonstrate water deficit conditions (Dunnett and Nolan, 2004). The composition and characteristics of green roofs depend on many factors. 
With the tremendous upsurge of interest in increasing the diversity of plant species in green roof systems, the edible plants were used extensively in recent year (Butler et al., 2012). It is also important to choose an appropriate substrate in which plants demonstrate drought-tolerant growth and can withstand the harsh rooftop environment.

Agastache rugosa is a perennial herb native to Korea, China and Japan that can grow to a height of 100-150 cm with quadrangular stems, displaying mauve or purple - blue flowers between June and September (Schafer, 2011). Agastache rugose thrives in warm and humid weather. It does grow best in full sun and well-drained sandy loam rich in organic matter, but also tolerate under somewhat poor soil and dry conditions. It can survive winter temperatures of at least $-10{ }^{\circ} \mathrm{C}$ without protection. It can propagate by seeds in the spring and autumn, as well as with rooted cutting (Yamani et al., 2014). The flowers attract various species of butterflies and are also known as a valuable source of nectar for honeybees and native bees. It is also used in traditional Chinese medicine to treat cholera, vomiting, fever, headaches, colds, vomiting, nausea, and stomach problems, as well as in other eastern Asia countries (Choi and Seo, 2007).

Hydrophilic polymers (hydrogel), able to hold water several hundred times their weight, are widely used to improve the moisture of soil in the field of agriculture, horticulture and forestry (Baran et al., 2015). Since the 2000's, studies have been conducted on the application of hydrophilic polymers in green roofs, along with the development of high interest in green roofs. Hydrophilic polymers are used in green roof substrates to enhance the water holding capacity and improve the growth of plants. Young et al. (2017) revealed that coarser particle size substrates amended with $1 \%(\mathrm{v} / \mathrm{v})$ dry hydrophilic polymer can significantly improve the drought tolerance of green roof plants, such as Festuca ovina and Linaria vulgaris. The growth of Salvia officinalis in $8 \mathrm{~cm}$ deep substrate mixed with hydrophilic polymer at a rate of $0.6 \%$ was the best under drought stress (Savi et al., 2014). Farrell et al. (2013) examined the impact of hydrophilic polymer addition to two green roof substrates, scoria-based and crushed terracotta roof-tile based, on the growth of winter wheat (Triticum aestivum L.) and white lupin (Lupinus albus L.). The results suggest that hydrogel addition can improve the plant available water, but had no effect on increasing wilting time. Olszewski et al. (2010) reported that the use of hydrophilic polymers increased the total porosity and container capacity of the substrate. The study also showed that hydrophilic polymer addition improved the initial plant growth of Sedum floriferum and Sedum spurium. Oschmann et al. (2007) conducted a three-year experiment with extensive green roof systems using crushed expanded slate substrates with 12 species of wild plants. The results revealed that use of hydrophilic polymers can enhance the water holding capacity and plant available water of substrates. In addition, the plants in substrates showed the best growth, especially at a concentration of $3 \mathrm{~g} \cdot \mathrm{L}^{-1}$, which resulted in the best coverage and regeneration. Meanwhile, our previous study found that the use of hydrophilic polymers in peat moss-based substrates significantly increased the substrate water content, but adversely affected plant growth, flowering and the visual quality of the drought-tolerant plant Aster koraiensis under no irrigation conditions (Xu et al., 2014). The response to hydrophilic polymers will thus depend upon the specific plant species and environmental conditions in the area. The objectives of this study were to determine the effect of different levels of water-retentive, hydrophilic polymer in three types of substrates on the growth, flower development and overwintering of Agastache rugosa in green roofs during the dry as well as rainy season. 


\section{Material and methods}

\section{Substrate preparation}

A total of 45 square containers $((50 \mathrm{~cm}(\mathrm{~L}) \times 50 \mathrm{~cm}(\mathrm{~W}) \times 25 \mathrm{~cm}(\mathrm{H}))$ were installed on the flat rooftop of the Complex Practice Building in Konkuk University, Chungju, Chungcheongbuk-do, South Korea located at latitude $35^{\circ} 49^{\prime} \mathrm{N}$ and longitude $127^{\circ} 08^{\prime} \mathrm{E}$ (Fig. 1). Monthly precipitation was about 88.2, 110.6, 277.7, 122.7, and $153.8 \mathrm{~mm}$ in June, July, August, September, and October, respectively, with very little precipitation in May and November. Diurnal maximum air temperature was $35.3{ }^{\circ} \mathrm{C}, 31.7{ }^{\circ} \mathrm{C}$, $34.4^{\circ} \mathrm{C}, 34.4^{\circ} \mathrm{C}, 31.0^{\circ} \mathrm{C}, 27.2^{\circ} \mathrm{C}$, and $17.5^{\circ} \mathrm{C}$ in May, June, July, August, September, October, and November, respectively. Minimum air temperature was $4.6{ }^{\circ} \mathrm{C}, 15.1{ }^{\circ} \mathrm{C}$, $17.0{ }^{\circ} \mathrm{C}, 17.4{ }^{\circ} \mathrm{C}, 9.5^{\circ} \mathrm{C}, 1.6^{\circ} \mathrm{C}$, and $-4.5^{\circ} \mathrm{C}$, respectively.

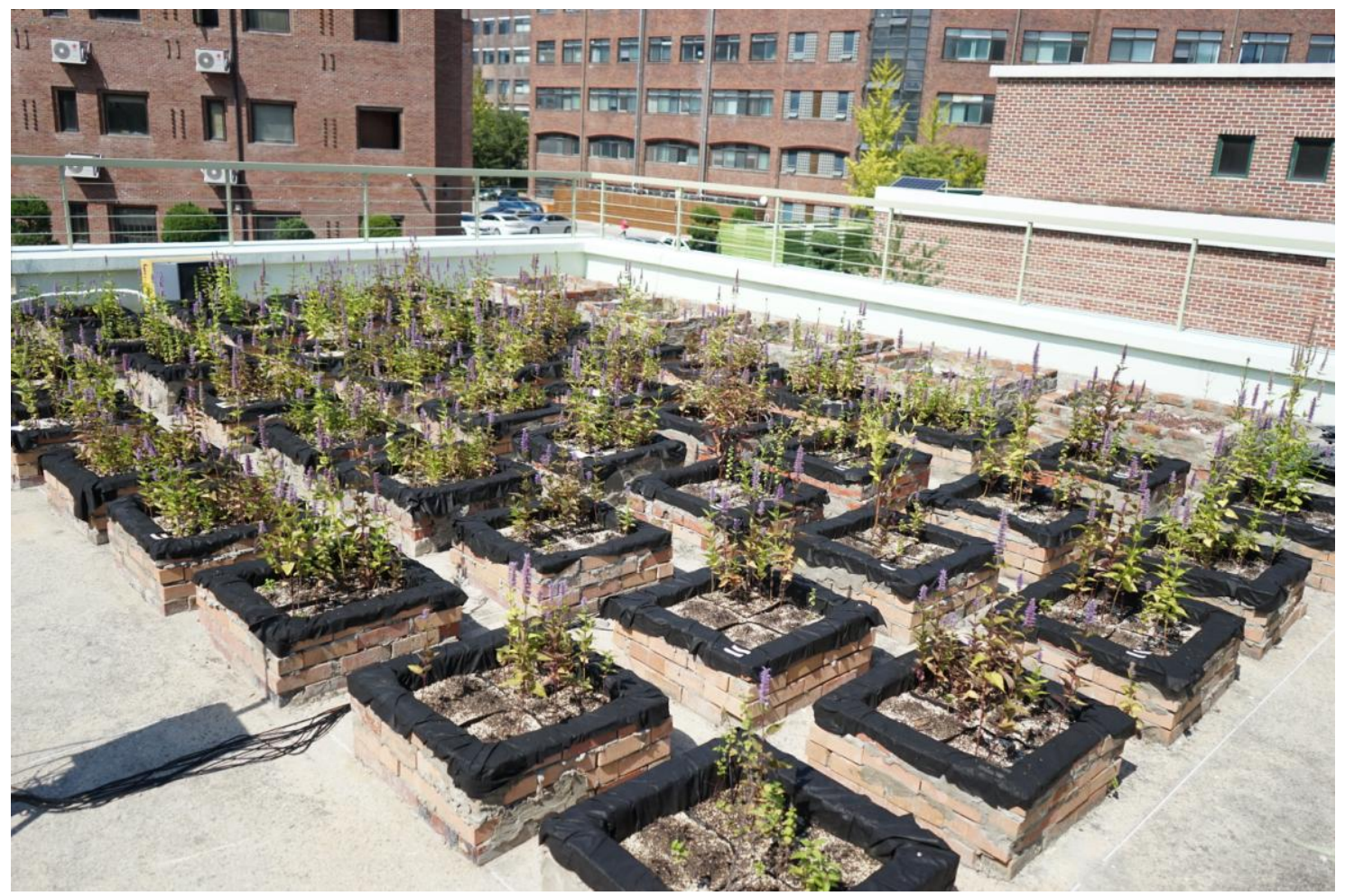

Figure 1. Experimental blocks on flat rooftop of the Complex Practice Building in Konkuk University, Chungju, Chungcheongbuk-do, South Korea

From top to bottom, each container was filled with the following four layers: vegetation, growing medium, geotextile filter, and plastic drainage. A geotextile filter with good air permeability and water permeability was used to filter the substrate and separate it from the drainage layer. Plastic drainage was used to support the three layers above, to cut off roots, increase insulation, and retain excess water (Papafotiou et al., 2013). Based on the growth habits of Agastache rugosa and previous studies on green roof substrates, experimental substrates were formulated consisting of 80,20 , or $50 \%$ (by volume) coir and 20, 80, or $50 \%$ (by volume) perlite. Hydrophilic polymer (KSAM, Kolon Chemical Co., Ltd., Korea) was incorporated into the substrates at concentrations of 0 (referred as control), $0.25,0.5,1.0$, or $2.0 \mathrm{~kg} \cdot \mathrm{m}^{-3}$ (polymer: medium $(\mathrm{w} / \mathrm{v})$, dry weight basis) with three replicates per treatment. Organic fertilizers were 
added to the substrates at $5 \%$ total volume to ensure that the same quantity was applied in each substrate which depth was $20 \mathrm{~cm}$.

\section{Plant material}

Seedlings of Agastache rugosa were obtained from a commercial nursery, grown on $10 \mathrm{~cm}$-diameter pots. Three seedlings of Agastache rugosa with consistent heights of $20 \mathrm{~cm}$ were transplanted into green roof containers on the 14th of May 2014. The initial height of the transplants differed by $<1 \mathrm{~cm}$. All plants were watered every 2 days in the first week followed by no watering when they were considered well established.

\section{Measurement of volumetric water content of the substrate and plant growth}

The volumetric water content in the top $10 \mathrm{~cm}$ of the substrates were monitored using a portable soil humidity tester (DM-5, Takemura ${ }^{\circledR}$, Japan), respectively, at four locations selected randomly. Three replications were performed at 5 p.m. every week during the experimental period. Meteorological data were recorded every $30 \mathrm{~min}$ using an automatic weather station (Sensus, Casella Group Ltd., UK).

The growth parameters observed were plant height, leaf number of all the plants $(\mathrm{n}=$ 9) in June (dry season) and August (rainy season), respectively. Plant height (H) above the stem base, widest width at the widest vegetative point of the plant passing through the center (W1), and widest width perpendicular to W1 (W2) were measured. The data for the height and width were used to calculate the growth index (GI) $([\mathrm{W} 1+\mathrm{W} 2] / 2+$ $\mathrm{H}) / 2$, commonly used as an indicator of plant size (Hammond et al., 2007). The relative appearance of the plant was evaluated based on its visual quality. The visual quality evaluations were divided into five grades, with grade 1 standing for severely stressed and completely dried out, grade 2 for stressed with less than $50 \%$ of the leaves retaining green pigmentation, grade 3 for mildly stressed with $50 \%$ of the leaves retaining green pigmentation, grade 4 for minor stress with over $50 \%$ of the leaves appearing to be healthy, and grade 5 for unstressed with all leaves appearing healthy (Nagase and Dunnett, 2010).

Leaf color was measured using a Chroma meter (CR-400, Konica Minolta Group, Japan) with $L^{*}, a^{*}$, and $b^{*}$ mode. The $L^{*}$ values ranged from black $\left(L^{*}=0\right)$ to white $\left(\mathrm{L}^{*}=100\right)$. The $\mathrm{a}^{*}$ values ranged from red $\left(\mathrm{a}^{*}=100\right)$ to green $\left(\mathrm{a}^{*}=-100\right)$. The $\mathrm{b}^{*}$ values ranged from yellow $\left(b^{*}=100\right)$ to blue $\left(b^{*}=-100\right)$. Chroma $C^{*}$ was calculated as $\left(a^{*} 2+b^{*} 2\right)^{1 / 2}$ measures for color saturation or intensity. Hue angle $h$ was calculated as $\tan ^{-1}\left(b^{*} / a^{*}\right)$. When $a^{*}$ was less than 0 and $b^{*}$ was greater than $0, h$ was $180+\tan ^{-1}\left(b^{*}\right.$ / $\left.\mathrm{a}^{*}\right)$. The value of $\mathrm{h}$ is a $360^{\circ}$ color wheel, with $0^{\circ}, 90^{\circ}, 180^{\circ}$, and $270^{\circ}$ representing red-purple, yellow, bluish-green, and blue, respectively (Wang and Camp, 2000). The leaves measured were the third from the top to the bottom of the plant. The Leaf color, chlorophyll contents of leaves and number of inflorescences were measured in August when plants were at peak flower time.

The chlorophyll meter (SPAD-502) is a simple tool used to measure the relative chlorophyll content or greenness, thus is an efficient indicator of stress in plants (Netto et al., 2005). The chlorophyll contents were measured for 9 leaves per container using a SPAD-502 meter (Minolta Camera Co., LTd, Osaka, Japan).

All the herb plants in green roofs overwinter with no protection. Overwintering survival rates and self-sown seeding number for all plants were recorded in May and 
June 2015 by measuring the number of sprouts aboveground from the roots and growing seedlings from seeds, respectively.

\section{Experiment design and statistical analysis}

This experiment was a completely randomized design with two factor, substrate design and hydrophilic polymer rate. There were three replicates for each treatment and each replicate consisted of three plants. Data were subjected to analysis of variance (ANOVA) with SAS 9.1 software package (SAS version 9.1, SAS Institute, Cary, NC). Significant mean separation was indicated by Duncan's multiple range test. Statistical significance was considered when the $\mathrm{p}$ value was less than 0.05 .

\section{Results}

\section{Volumetric water content of substrate}

Under $\mathrm{C}_{4} \mathrm{P}_{1}$ treatment, the average volumetric water content was 52.7, 60.3, 61.3, 66.5 , or $85.3 \%$ with 0 (control), $0.25,0.5,1.0$, or $2.0 \mathrm{~kg} \cdot \mathrm{m}^{-3}$ of hydrophilic polymer, respectively. Under $\mathrm{C}_{1} \mathrm{P}_{4}$ treatment, it was $14.0,14.1,14.4,17.8$, or $25.3 \%$, respectively, and $48.9,50.4,53.4,58.5$, or $82.6 \%$ under $\mathrm{C}_{1} \mathrm{P}_{1}$ treatment (Fig. 2). The moisture contents of substrate $\mathrm{C}_{4} \mathrm{P}_{1}$ and $\mathrm{C}_{1} \mathrm{P}_{1}$ under the control treatment were almost 3.8- and 3.5- fold higher than that of substrate $\mathrm{C}_{1} \mathrm{P}_{4}$, respectively. There was a significant $(p<0.001)$ interactive effect of the substrate and the hydrophilic polymer content on the volumetric water content. The temperatures of the substrates were significantly affected by treatment with hydrophilic polymer during the study (data not shown). The temperature of the substrate dropped significantly $(p<0.001)$ as the hydrophilic polymer content in the substrate was increased.

\section{Plant growth}

As shown in Table 1, the growth index of Agastache rugosa was not significantly different among the three substrates when measured in June. However, the leaf number $(p=0.009)$ and visual rating $(p=0.028)$ were significantly greater in substrate $\mathrm{C}_{1} \mathrm{P}_{4}$ than in substrate $\mathrm{C}_{4} \mathrm{P}_{1}$ in August. In addition, the growth index $(p<0.001)$, leaf number $(p<0.001)$ and visual rating $(p<0.001)$ of Agastache rugosa were significantly greater in substrate $\mathrm{C}_{1} \mathrm{P}_{4}$ than those in other two substrates when they were measured.

\section{Flower development}

The purple-blue flowers of Agastache rugosa started to bloom from mid-July to midOctober, with a flowering period extending more than 90 days. The peak blooming period was from mid-August to early September. The total number of inflorescences per Agastache rugosa plant was significantly $(p=0.002)$ higher in $\mathrm{C}_{1} \mathrm{P}_{4}$ than in the other substrates (Fig. 3). Incorporation of $1.0 \mathrm{~kg} \cdot \mathrm{m}^{-3}$ hydrophilic polymer significantly increased the total number of inflorescences per Agastache rugosa plant in $\mathrm{C}_{1} \mathrm{P}_{4}$, which was more than 2- fold higher than those in either the control or other hydrophilic polymer treatments. However, there was no significant difference in the total number of inflorescences per plant between the control and hydrophilic polymer treatment in $\mathrm{C}_{4} \mathrm{P}_{1}$ and $\mathrm{C}_{1} \mathrm{P}_{1}$. 


$$
-5508-
$$
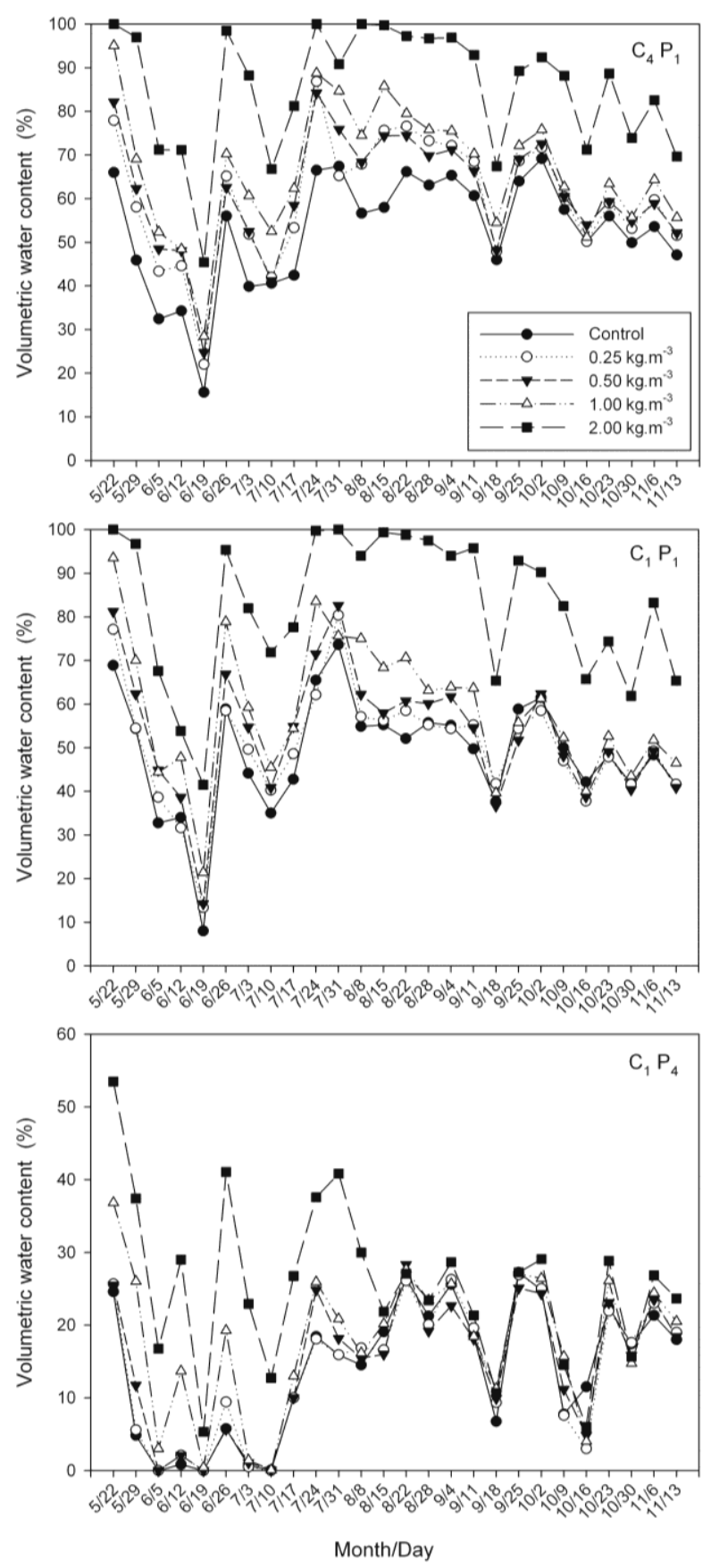

Figure 2. Volumetric water content of three different green roof substrates $\left(C_{4} P_{1}\right.$ : coir $80 \%$, perlite $20 \%$; $C_{1} P_{1}$ : coir $50 \%$, perlite $50 \%$; $C_{l} P_{4}$ : coir $20 \%$, perlite $80 \%(\%$ by vol.)) containing different amounts of hydrophilic polymer: 0 (Control), 0.25, 0.5, 1.0, and $2.0 \mathrm{~kg} \cdot \mathrm{m}^{-3}$ (polymer: medium ( $w / v)$, dry weight basis) 
Table 1. Growth index, leaf number and visual rating of Agastache rugosa grown in different green roof substrates during the dry season (June) and the rainy season (August)

\begin{tabular}{|c|c|c|c|c|c|c|c|}
\hline \multirow[b]{2}{*}{ Substrate } & \multirow[b]{2}{*}{$\begin{array}{l}\text { Polymer rate } \\
\left(\mathbf{k g} \cdot \mathrm{m}^{-3}\right)\end{array}$} & \multicolumn{3}{|c|}{ June (dry season) } & \multicolumn{3}{|c|}{ August (rainy season) } \\
\hline & & $\begin{array}{l}\text { Growth } \\
\text { index }\end{array}$ & $\begin{array}{c}\text { Leaf } \\
\text { number } \\
\text { (per plant) }\end{array}$ & $\begin{array}{l}\text { Visual } \\
\text { rating }\end{array}$ & $\begin{array}{l}\text { Growth } \\
\text { index }\end{array}$ & $\begin{array}{c}\text { Leaf } \\
\text { number } \\
\text { (per plant) }\end{array}$ & $\begin{array}{l}\text { Visual } \\
\text { rating }\end{array}$ \\
\hline \multirow{5}{*}{$\mathrm{C}_{4} \mathrm{P}_{1}^{\mathrm{y}}$} & Control $^{\mathrm{X}}$ & $17.50 \mathrm{ab}^{\mathrm{z}}$ & $13.1 \mathrm{~b}$ & $3.8 \mathrm{~b}$ & $20.09 \mathrm{bc}$ & $12.0 \mathrm{c}$ & $2.2 \mathrm{~cd}$ \\
\hline & 0.25 & $16.60 \mathrm{~b}$ & 14.6ab & 3.9ab & $20.43 b c$ & $14.4 \mathrm{bc}$ & $2.3 \mathrm{c}$ \\
\hline & 0.5 & $16.90 \mathrm{ab}$ & $16.6 \mathrm{ab}$ & 4.3ab & $19.65 \mathrm{c}$ & $8.7 \mathrm{c}$ & $2.3 \mathrm{c}$ \\
\hline & 1.0 & $17.39 \mathrm{ab}$ & $16.8 \mathrm{ab}$ & $4.1 \mathrm{ab}$ & $22.99 \mathrm{a}-\mathrm{c}$ & $12.3 \mathrm{c}$ & $2.4 \mathrm{c}$ \\
\hline & 2.0 & $17.82 \mathrm{ab}$ & $11.1 \mathrm{~b}$ & $3.9 \mathrm{ab}$ & $21.09 b c$ & $10.3 \mathrm{c}$ & $1.6 \mathrm{~d}$ \\
\hline \multirow{6}{*}{$\mathrm{C}_{1} \mathrm{P}_{1}$} & Control & $19.44 a$ & $22.1 \mathrm{a}$ & $3.9 \mathrm{ab}$ & $24.75 a-c$ & $13.3 \mathrm{bc}$ & $2.6 \mathrm{bc}$ \\
\hline & 0.25 & $17.56 \mathrm{ab}$ & $15.4 \mathrm{ab}$ & 4.2ab & $19.53 \mathrm{c}$ & $10.7 \mathrm{c}$ & $2.8 \mathrm{bc}$ \\
\hline & 0.5 & $17.68 \mathrm{ab}$ & $17.3 \mathrm{ab}$ & $4.1 \mathrm{ab}$ & $22.29 \mathrm{a}-\mathrm{c}$ & $14.4 \mathrm{bc}$ & $2.8 \mathrm{bc}$ \\
\hline & 1.0 & 17.60ab & $19.6 \mathrm{ab}$ & $4.1 \mathrm{ab}$ & $24.77 \mathrm{a}-\mathrm{c}$ & $14.4 \mathrm{bc}$ & $2.9 \mathrm{~b}$ \\
\hline & 2.0 & $17.31 \mathrm{ab}$ & $17.4 a b$ & $3.9 \mathrm{ab}$ & $20.09 b c$ & $9.7 \mathrm{c}$ & $2.3 \mathrm{c}$ \\
\hline & Control & $19.31 \mathrm{ab}$ & $18.8 \mathrm{ab}$ & $4.1 \mathrm{ab}$ & $28.43 a$ & $22.9 \mathrm{a}$ & $4.0 \mathrm{ab}$ \\
\hline \multirow{3}{*}{$\mathrm{C}_{1} \mathrm{P}_{4}$} & 0.25 & $17.10 \mathrm{ab}$ & $16.3 \mathrm{ab}$ & $4.2 \mathrm{ab}$ & $25.12 \mathrm{a}-\mathrm{c}$ & $25.7 \mathrm{a}$ & $4.1 \mathrm{ab}$ \\
\hline & 0.5 & $18.53 \mathrm{ab}$ & $22.4 \mathrm{a}$ & $4.3 \mathrm{ab}$ & $28.16 \mathrm{a}$ & $26.4 \mathrm{a}$ & $4.2 \mathrm{ab}$ \\
\hline & 1.0 & 18.10ab & $19.6 \mathrm{ab}$ & $4.4 \mathrm{a}$ & $27.86 \mathrm{a}$ & $20.7 \mathrm{ab}$ & $4.4 \mathrm{a}$ \\
\hline \multirow{4}{*}{ Significance } & 2.0 & $17.46 \mathrm{ab}$ & $17.1 \mathrm{ab}$ & $4.4 \mathrm{a}$ & 26.39ab & $20.3 \mathrm{ab}$ & $3.8 \mathrm{~b}$ \\
\hline & Substrate & NS & $* *$ & $*$ & $* * *$ & $* * *$ & $* * *$ \\
\hline & Polymer rate & NS & NS & NS & NS & NS & $*$ \\
\hline & Interaction & NS & NS & NS & NS & NS & NS \\
\hline
\end{tabular}

$* * *, * *, *: p<0.001, p<0.01, p<0.05$, respectively. NS: not significant

${ }^{x}$ Hydrophilic polymer rate: 0 (referred as control), $0.25,0.5,1.0$, and $2.0 \mathrm{~kg} \cdot \mathrm{m}^{-3}$ [polymer: medium $(\mathrm{w} / \mathrm{v})$, dry weight basis]

${ }^{y}$ Substrate: $\mathrm{C}_{4} \mathrm{P}_{1}$ : coir $80 \%$, perlite $20 \% ; \mathrm{C}_{1} \mathrm{P}_{1}$ : coir $50 \%$, perlite $50 \% ; \mathrm{C}_{1} \mathrm{P}_{4}$ : coir $20 \%$, perlite $80 \%(\%$ by volume)

${ }^{\mathrm{z}}$ Means within columns by substrate were determined by Duncan's multiple rang test at $p \leq 0.05$ level

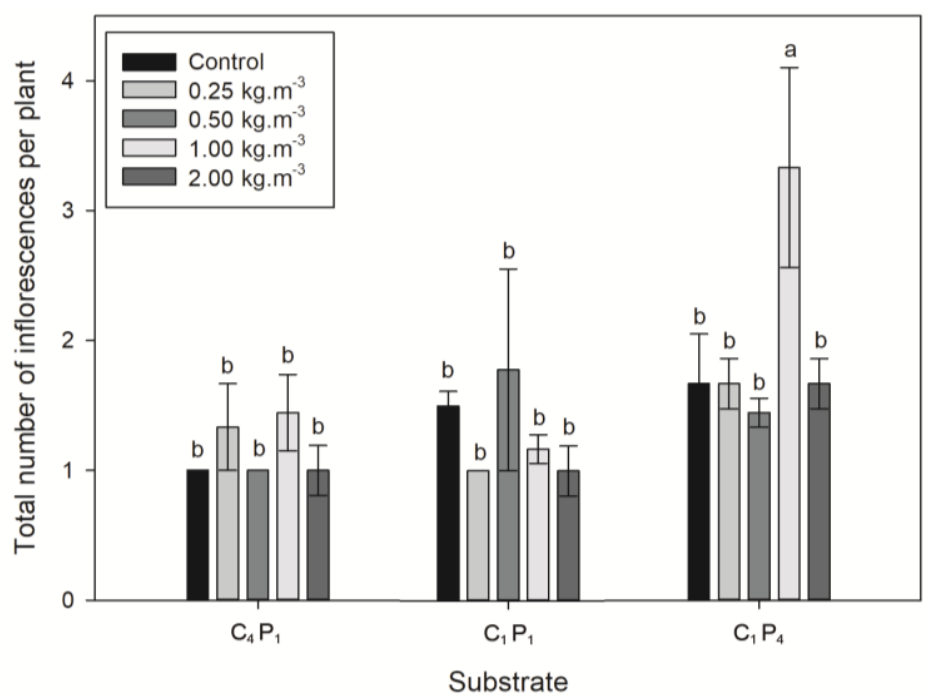

Figure 3. Effect of total number of inflorescences per plant for Agastache rugosa in the three different green roof substrates $\left(C_{4} P_{1}\right.$ : coir $80 \%$, perlite $20 \%$; $C_{1} P_{1}$ : coir $50 \%$, perlite $50 \%$;

$C_{1} P_{4}$ : coir $20 \%$, perlite $80 \%$ (\% by vol.)) containing different amounts of hydrophilic polymer: $O$ (Control), $0.25,0.5,1.0$, and $2.0 \mathrm{~kg} \cdot \mathrm{m}^{-3}$ (polymer: medium ( $\left.w / \mathrm{v}\right)$, dry weight basis) 
As shown in Table 2, there was an interactive effect between the substrate and the hydrophilic polymer on lightness $\left(\mathrm{L}^{*}\right)$ (interaction $p=0.010$ ), values of $\mathrm{a}^{*}$ (interaction $p$ $=0.003$ ), $\mathrm{b}^{*}$ (interaction $p=0.009$ ), Chroma $\left(\mathrm{C}^{*}\right)$ (interaction $p=0.007$ ) and hue angle (h) (interaction $p=0.040$ ) in August when plants were at the peak flowering stage. Lower values of $\mathrm{L}^{*}$ and $\mathrm{a}^{*}$ as well as higher hue angles were recorded in the leaves of plants in $\mathrm{C}_{1} \mathrm{P}_{4}$, confirming the darker green color of the foliage compared to $\mathrm{C}_{4} \mathrm{P}_{1}$ and $\mathrm{C}_{1} \mathrm{P}_{1}$. The leaves in $\mathrm{C}_{4} \mathrm{P}_{1}$ had a less vivid green color than those in $\mathrm{C}_{1} \mathrm{P}_{4}$ and $\mathrm{C}_{1} \mathrm{P}_{1}$. Treatment with $1.0 \mathrm{~kg} \cdot \mathrm{m}^{-3}$ hydrophilic polymer greatly increased the value of $\mathrm{L}^{*}$ (brighter), $\mathrm{b}^{*}$ (more yellow) and Chroma (more vivid), but decreased the value of a* (more green) in substrate $\mathrm{C}_{4} \mathrm{P}_{1}$. In $\mathrm{C}_{1} \mathrm{P}_{1}$, hydrophilic polymer amendment increased the value of $\mathrm{L}^{*}$ (brighter), $\mathrm{b}^{*}$ (more yellow), and $\mathrm{C}^{*}$ (more vivid), but decreased $\mathrm{a}^{*}$ values (more green) and $\mathrm{h}$ values (greener). The SPAD value for all treatments in $\mathrm{C}_{1} \mathrm{P}_{4}$ was better than for other substrates. Moreover, the leaf performances were the highest under $\mathrm{C}_{1} \mathrm{P}_{4}$ treatment when measured in August, with large leaf sizes and green healthy leaves (Fig. 4).

Table 2. Color measurements and SPAD values of Agastache rugosa grown in three different green roof substrates in August at the peak flowing time

\begin{tabular}{c|c|c|c|c|c|c|c}
\hline Substrate & $\begin{array}{c}\text { Polymer } \\
\text { rate }\left(\mathbf{k g} \cdot \mathbf{m}^{-3}\right)\end{array}$ & $\mathbf{L}^{*}$ & $\mathbf{a}^{*}$ & $\mathbf{b}^{*}$ & $\mathbf{C}^{*}$ & $\mathbf{h}$ & $\begin{array}{c}\text { SPAD } \\
\text { value }\end{array}$ \\
\hline \multirow{5}{*}{$\mathrm{C}_{4} \mathrm{P}_{1}{ }^{\mathrm{y}}$} & Control $^{\mathrm{x}}$ & $40.10 \mathrm{c}-\mathrm{f}^{\mathrm{z}}$ & $-2.51 \mathrm{a}-\mathrm{c}$ & $23.85 \mathrm{c}-\mathrm{f}$ & $24.59 \mathrm{~b}-\mathrm{c}$ & $91.56 \mathrm{ab}$ & $17.13 \mathrm{~d}$ \\
& 0.25 & $35.22 \mathrm{~d}-\mathrm{f}$ & $-2.65 \mathrm{a}-\mathrm{c}$ & $19.10 \mathrm{~d}-\mathrm{f}$ & $19.91 \mathrm{~cd}$ & $91.98 \mathrm{ab}$ & $20.76 \mathrm{~cd}$ \\
& 0.5 & $52.52 \mathrm{a}$ & $-17.79 \mathrm{e}$ & $42.07 \mathrm{a}$ & $45.68 \mathrm{a}$ & $112.92 \mathrm{a}$ & $20.06 \mathrm{~cd}$ \\
& 1.0 & $36.90 \mathrm{c}-\mathrm{f}$ & $-6.59 \mathrm{~b}-\mathrm{d}$ & $20.19 \mathrm{c}-\mathrm{f}$ & $21.24 \mathrm{~cd}$ & $108.08 \mathrm{a}$ & $23.11 \mathrm{bc}$ \\
& 2.0 & $32.79 \mathrm{f}$ & $2.50 \mathrm{ab}$ & $13.46 \mathrm{f}$ & $16.22 \mathrm{~d}$ & $65.74 \mathrm{~b}$ & $19.48 \mathrm{~cd}$ \\
& Control & $34.59 \mathrm{ef}$ & $6.28 \mathrm{a}$ & $14.45 \mathrm{ef}$ & $16.80 \mathrm{~d}$ & $62.19 \mathrm{~b}$ & $19.77 \mathrm{~cd}$ \\
$\mathrm{C}_{1} \mathrm{P}_{1}$ & 0.25 & $44.84 \mathrm{a}-\mathrm{d}$ & $-11.42 \mathrm{c}-\mathrm{e}$ & $29.59 \mathrm{a}-\mathrm{d}$ & $31.91 \mathrm{a}-\mathrm{c}$ & $108.84 \mathrm{a}$ & $24.16 \mathrm{bc}$ \\
& 0.5 & $50.42 \mathrm{a}$ & $-18.38 \mathrm{e}$ & $38.60 \mathrm{ab}$ & $42.75 \mathrm{a}$ & $115.46 \mathrm{a}$ & $23.80 \mathrm{bc}$ \\
& 1.0 & $51.34 \mathrm{ab}$ & $-18.87 \mathrm{e}$ & $40.38 \mathrm{ab}$ & $44.57 \mathrm{a}$ & $115.07 \mathrm{a}$ & $26.14 \mathrm{~b}$ \\
& 2.0 & $46.48 \mathrm{a}-\mathrm{c}$ & $-12.72 \mathrm{de}$ & $34.15 \mathrm{a}-\mathrm{c}$ & $36.44 \mathrm{ab}$ & $110.43 \mathrm{a}$ & $20.53 \mathrm{~cd}$ \\
& Control & $41.47 \mathrm{~b}-\mathrm{f}$ & $-16.73 \mathrm{e}$ & $27.67 \mathrm{~b}-\mathrm{e}$ & $32.34 \mathrm{a}-\mathrm{c}$ & $121.20 \mathrm{a}$ & $30.71 \mathrm{a}$ \\
& 0.25 & $43.55 \mathrm{a}-\mathrm{e}$ & $-17.34 \mathrm{e}$ & $31.12 \mathrm{a}-\mathrm{d}$ & $35.63 \mathrm{ab}$ & $119.24 \mathrm{a}$ & $30.99 \mathrm{a}$ \\
$\mathrm{C}_{1} \mathrm{P}_{4}$ & 0.5 & $44.47 \mathrm{a}-\mathrm{d}$ & $-16.78 \mathrm{e}$ & $32.14 \mathrm{a}-\mathrm{d}$ & $36.26 \mathrm{ab}$ & $117.68 \mathrm{a}$ & $34.94 \mathrm{a}$ \\
& 1.0 & $41.18 \mathrm{~b}-\mathrm{f}$ & $-16.45 \mathrm{e}$ & $27.95 \mathrm{a}-\mathrm{e}$ & $32.46 \mathrm{a}-\mathrm{c}$ & $120.52 \mathrm{a}$ & $35.06 \mathrm{a}$ \\
& 2.0 & $44.34 \mathrm{a}-\mathrm{d}$ & $-17.23 \mathrm{e}$ & $32.61 \mathrm{a}-\mathrm{d}$ & $36.90 \mathrm{ab}$ & $117.78 \mathrm{a}$ & $31.20 \mathrm{a}$ \\
Significance & Substrate & NS & $* *$ & $*$ & $* *$ & $* *$ & $* * *$ \\
& Polymer rate & $* *$ & $* *$ & $* *$ & $* *$ & NS & $* * *$ \\
& Interaction & $* *$ & $* *$ & $* *$ & $* *$ & $*$ & NS \\
\hline
\end{tabular}

$* * *, * *, *: p<0.001, p<0.01, p<0.05$, respectively. NS: not significant

${ }^{x}$ Hydrophilic polymer rate: 0 (referred as control), $0.25,0.5,1.0$, and $2.0 \mathrm{~kg} \cdot \mathrm{m}^{-3}$ [polymer: medium $(\mathrm{w} / \mathrm{v})$, dry weight basis]

${ }^{\mathrm{y}}$ Substrate: $\mathrm{C}_{4} \mathrm{P}_{1}$ : coir $80 \%$, perlite $20 \% ; \mathrm{C}_{1} \mathrm{P}_{1}$ : coir $50 \%$, perlite $50 \% ; \mathrm{C}_{1} \mathrm{P}_{4}$ : coir $20 \%$, perlite $80 \%(\%$ by volume)

${ }^{\mathrm{z}}$ Means within columns by substrate were determined by Duncan's multiple rang test at $p<0.05$ level

\section{Overwintering}

There was an interactive effect between substrate and hydrophilic polymer rate on overwintering rate (interaction $p=0.041$ ) and self-sown seedling number (interaction $p<0.001$ ) of Agastache rugosa, with significantly higher overwintering rate and selfsown seedling number in substrate $\mathrm{C}_{1} \mathrm{P}_{4}$ than that in $\mathrm{C}_{4} \mathrm{P}_{1}$ and $\mathrm{C}_{1} \mathrm{P}_{1}$ (Table 3). In 
substrate $\mathrm{C}_{1} \mathrm{P}_{4}$, the addition of hydrophilic polymer decreased the overwintering rate, especially at rate of $2.0 \mathrm{~kg} \cdot \mathrm{m}^{-3}$. However, hydrophilic polymer increased self-sown seedling number of Agastache rugosa in substrate $\mathrm{C}_{1} \mathrm{P}_{4}$, especially at the rate of 1.0 and $2.0 \mathrm{~kg} \cdot \mathrm{m}^{-3}$ compared to other hydrophilic polymer treatments or control. While in substrate $\mathrm{C}_{4} \mathrm{P}_{1}$ and $\mathrm{C}_{1} \mathrm{P}_{1}$, self-sown seedling number decreased by hydrophilic polymer treatment.

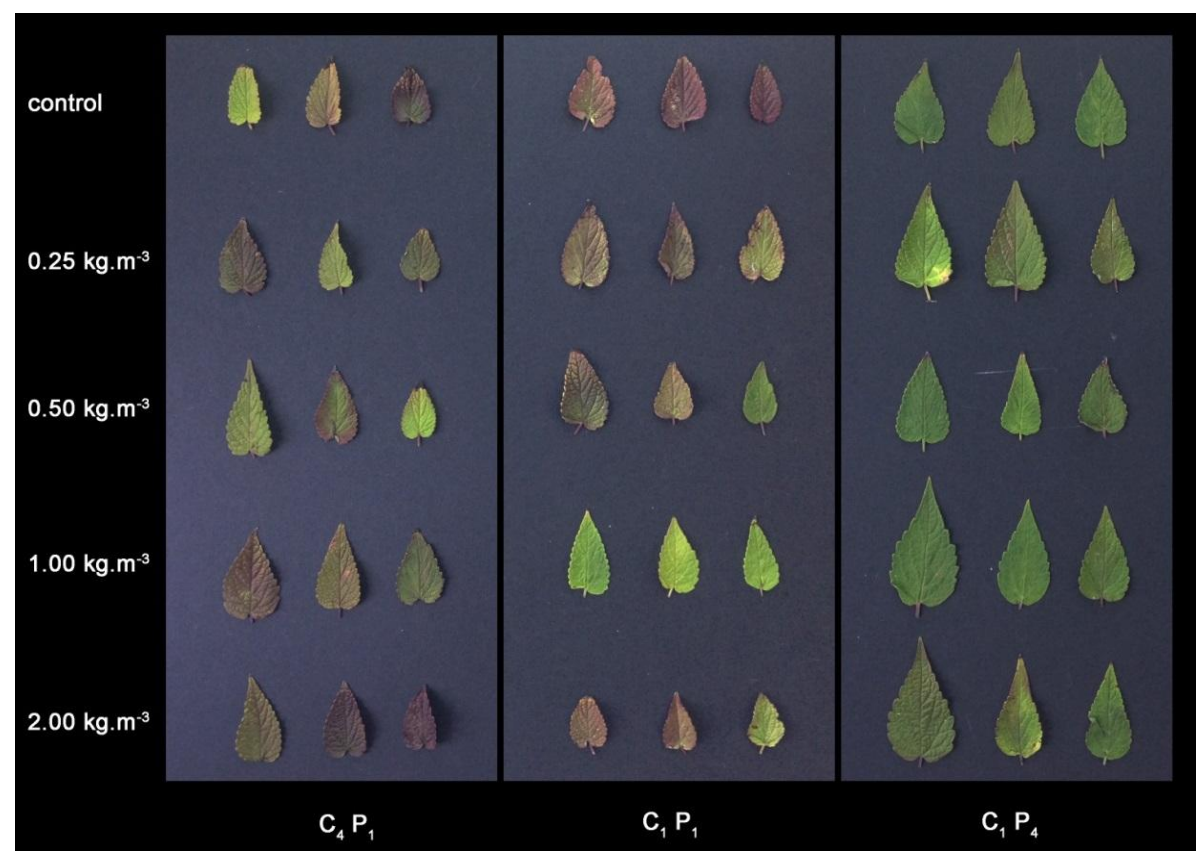

Figure 4. Leaf performance for Agastache rugosa in the three different green roof substrates $\left(C_{4} P_{1}\right.$ : coir $80 \%$, perlite $20 \% ; C_{1} P_{1}:$ coir $50 \%$, perlite $50 \% ; C_{1} P_{4}:$ coir $20 \%$, perlite $80 \%(\%$ by vol.)) containing different amounts of hydrophilic polymer: 0 (Control), 0.25, 0.5, 1.0, and 2.0 $\mathrm{kg} \cdot \mathrm{m}^{-3}$ (polymer: medium (w/v), dry weight basis) in August at peak blooming time

Table 3. Overwintering rate and self-sown seeding number of Agastache rugosa grown in three different green roof substrates

\begin{tabular}{c|c|c|c|c|c|c}
\hline \multirow{2}{*}{$\begin{array}{c}\text { Polymer rate } \\
\left(\mathbf{k g} \cdot \mathbf{m}^{-\mathbf{3}} \mathbf{)}\right.\end{array}$} & \multicolumn{3}{|c|}{ Overwintering rate $\mathbf{( \% )}$} & \multicolumn{3}{c}{ Self-sown seedling number } \\
\cline { 2 - 7 } & $\mathbf{C}_{\mathbf{4}} \mathbf{P}_{\mathbf{1}}^{\mathbf{y}}$ & $\mathbf{C}_{\mathbf{1}} \mathbf{P}_{\mathbf{1}}$ & $\mathbf{C}_{\mathbf{1}} \mathbf{P}_{\mathbf{4}}$ & $\mathbf{C}_{\mathbf{4}} \mathbf{P}_{\mathbf{1}}$ & $\mathbf{C}_{\mathbf{1}} \mathbf{P}_{\mathbf{1}}$ & $\mathbf{C}_{\mathbf{1}} \mathbf{P}_{\mathbf{4}}$ \\
\hline Control $^{\mathrm{x}}$ & $33.3 \mathrm{~d}^{\mathrm{z}}$ & $33.3 \mathrm{~d}$ & $100.0 \mathrm{a}$ & $22.3 \mathrm{de}$ & $30 \mathrm{~d}$ & $49.0 \mathrm{c}$ \\
0.25 & $55.6 \mathrm{~cd}$ & $50.0 \mathrm{~cd}$ & $77.8 \mathrm{a}-\mathrm{c}$ & $20.3 \mathrm{~d}-\mathrm{f}$ & $22.0 \mathrm{de}$ & $57.3 \mathrm{bc}$ \\
0.5 & $33.3 \mathrm{~d}$ & $66.7 \mathrm{~b}-\mathrm{d}$ & $88.9 \mathrm{ab}$ & $12.0 \mathrm{e}-\mathrm{g}$ & $15.0 \mathrm{~d}-\mathrm{g}$ & $65.0 \mathrm{~b}$ \\
1.0 & $44.4 \mathrm{~d}$ & $50.0 \mathrm{~cd}$ & $77.8 \mathrm{a}-\mathrm{c}$ & $13.5 \mathrm{e}-\mathrm{g}$ & $13.5 \mathrm{e}-\mathrm{g}$ & $90.5 \mathrm{a}$ \\
2.0 & $33.3 \mathrm{~d}$ & $33.3 \mathrm{~d}$ & $55.6 \mathrm{~cd}$ & $4.0 \mathrm{~g}$ & $6.0 \mathrm{gf}$ & $80.5 \mathrm{a}$ \\
Significant & & & & & & \\
Substrate & $* * *$ & & & $* * *$ & & \\
Polymer rate & $\mathrm{NS}$ & & & $\mathrm{NS}$ & & \\
Interaction & $*$ & & & $* * *$ & & \\
\hline
\end{tabular}

$* * *, * *, *: p<0.001, p<0.01, p<0.05$, respectively. NS: not significant

${ }^{\mathrm{x}}$ Hydrophilic polymer rate: 0 (referred as control), $0.25,0.5,1.0$, and $2.0 \mathrm{~kg} \cdot \mathrm{m}^{-3}$ [polymer: medium $(\mathrm{w} / \mathrm{v})$, dry weight basis]

${ }^{\mathrm{y}}$ Substrate: $\mathrm{C}_{4} \mathrm{P}_{1}$ : coir $80 \%$, perlite $20 \% ; \mathrm{C}_{1} \mathrm{P}_{1}$ : coir $50 \%$, perlite $50 \% ; \mathrm{C}_{1} \mathrm{P}_{4}$ : coir $20 \%$, perlite $80 \%(\%$ by volume)

${ }^{\mathrm{z}}$ Means within columns by substrate were determined by Duncan's multiple rang test at $p<0.05$ level 


\section{Discussion}

Our results revealed that more water was retained in the substrates when hydrophilic polymers were added compared to the control substrate. However, the amounts of additional volumetric water content in the three substrates depended on the compositions of the substrate. Hydrophilic polymer at $2.0 \mathrm{~kg} \cdot \mathrm{m}^{-3}$ significantly increased the average volumetric water content to values 1.62-, 1.81-, and 1.69- fold greater than that with no hydrophilic polymer in substrates $\mathrm{C}_{4} \mathrm{P}_{1}, \mathrm{C}_{1} \mathrm{P}_{4}$, and $\mathrm{C}_{1} \mathrm{P}_{1}$, respectively. In agreement with our study results, many studies have demonstrated that water retention in soil was increased with the addition of hydrophilic polymers, particularly in coarsetextured sandy soil (Dorraji et al., 2010). As the hydrophilic polymer content in the substrate increased, the substrate water content also increased, resulting in decreased temperature in the substrate (Nardini et al., 2012). The difference in volumetric water content between substrates with and without hydrophilic polymers decreased over time, especially in the coarse-textured substrate $\mathrm{C}_{1} \mathrm{P}_{4}$ starting from August. The difference also decreased with hydrophilic polymer concentrations less than $2.0 \mathrm{~kg} \cdot \mathrm{m}^{-3}$ in other substrate treatments. This reduction might be due to the loss of hydrophilic polymer effectiveness with time (Geesing and Schmidhalter, 2004). According to Han et al. (2013), the capacity of absorbing and desorbing water decreased with time and outside water condition. However, Jobin et al. (2004) found that there was no difference in available water and air-filled porosity in three substrates containing hydrophilic polymer after 9 weeks. Bai et al. (2010) studied the soil moisture of sandy soils mixed with four types of super-absorbent polymers under alternating dry and wet conditions. They found that the water retention capacity of the polymer decreased sharply when soil moisture was lower than a critical threshold. In addition, the water holding capacity of hydrophilic polymers could be affected by the extreme environmental conditions on the rooftop such as intensive solar radiation, high temperature, water deficits, freeze-thaw cycles caused by season change, and wetting-drying cycle due to weather change (Andry et al., 2009). In the present study, high temperature and frequent rainfall during the summer rainy season in August might have resulted in frequent wetting and drying of the substrates, leading to the loss of hydrophilic polymer effectiveness. This was associated with coarse-textured substrates, such as $\mathrm{C}_{1} \mathrm{P}_{4}$, that have a lower holding capacity, even with $2.0 \mathrm{~kg} \cdot \mathrm{m}^{-3}$ hydrophilic polymer.

Our study revealed that the $\mathrm{C}_{4} \mathrm{P}_{1}$ and $\mathrm{C}_{1} \mathrm{P}_{1}$ substrates, which have high coir dust content, are not ideal for Agastache rugosa in green roofs, either under drought stress or frequent rainfall. In our study, the moisture contents of substrate $\mathrm{C}_{4} \mathrm{P}_{1}$ and $\mathrm{C}_{1} \mathrm{P}_{1}$ under the control treatment were almost 3.8- and 3.5- fold higher than that of substrate $\mathrm{C}_{1} \mathrm{P}_{4}$, respectively. Although high coir dust content can increase the water content of substrates remarkably, it had no significant effect on plant growth for Agastache rugosa in June with little rainfall. Conversely, decreased leaf number and visual rate were observed in $\mathrm{C}_{4} \mathrm{P}_{1}$ with high coir dust content. In this study, the high coir dust content significantly decreased the number of inflorescences and ornamental quality in the peak flower period. Because of the high water-holding capacity, high organic content substrates are commonly used as container media, but are not recommended in green roofs in many studies (Cho et al., 2006). Beattie and Berghage (2004) suggested that substrates composed of $80-100 \%$ mineral matter and $0-20 \%$ organic matter suited for green roofs. Dunnett and Kingsburry (2004) also stated that low proportions of organic matter should be used, at between $10 \%$ and $20 \%$ of the total volume. The result is also consistent with the study findings that the substrate containing $10 \%$ organic matter was 
optimal for Allium schoenoprasum, Limonium latifolium, Melica ciliate and Nepeta $\times$ faassenii because of stable growth under dry and wet conditions in green roofs (Nagase and Dunnett, 2011). Bates et al. (2015) conducted a six-year experiment which showed that the substrate with low organic matter content was suitable for brown (biodiversity) roofs. In our study, the peak flower time for Agastache rugosa was the summer rainy season with high temperatures and frequent rainfall, which might have resulted in frequent wetting and drying of the substrates in the green roof. Increased coir dust content could result in lush growth that may suffer damage under drought stress or frequent rainfall, resulting in frequent drying and wetting. Rowe et al. (2006) found that high organic matter in substrates may result in shrinkage because of decomposition. They may also increase poor growth of Agastache rugosa in the substrates $\mathrm{C}_{4} \mathrm{P}_{1}$ and $\mathrm{C}_{1} \mathrm{P}_{1}$, with worse flowering and reddish purple leaves in August. In our study, the results showed that hydrophilic polymer addition had no significant effect on the growth of Agastache rugosa in June, during which plants were exposed to less rainfall, though the hydrophilic polymer significantly increased the water content of the substrates. The results indicated that Agastache rugosa has high drought tolerance that can withstand extended dry conditions on green roofs. Although mineral-based substrates are recommended in green roofs, water scarcity is typically the main problem because of extreme environmental conditions, even in the rainy season. The flowering is the most sensitive phase to water stress (Álvarez et al., 2013) and the effect of water deficits on flowering has been suggested in many studies. Koshita and Takahara (2004) found that water stress reduced the number of flowers produced by Citrus unshiu during flowerbud induction periods. Caser et al. (2017) showed that severe water stress significantly decreased flower number and delayed the flowering period of Primula vulgaris 'Heidy'. The importance of water stress was also examined by Razmjoo et al. (2008), who found that the number of flowers produced by Matricaria chamomile decreased with long interval irrigation. Similar effects of water stress on flower production have also been observed in crops. Passioura (2006) observed that water deficit greatly affected crop productivity, especially when it occurred at the flowering stage. The number of flowers and yield of Solanum melongena L. was markedly decreased when water regimes were adopted (Lovelli et al., 2007). In our study, hydrophilic polymer addition at a concentration of $1.0 \mathrm{~kg} \cdot \mathrm{m}^{-3}$ increased the water content in substrate $\mathrm{C}_{1} \mathrm{P}_{4}$, thereby improving the substrate condition and significantly increasing the number of flowers produced by Agastache rugosa.

The Agastache rugosa in substrate $\mathrm{C}_{1} \mathrm{P}_{4}$ had high rate of overwintering success with over $70 \%$, except in $2.0 \mathrm{~kg} \cdot \mathrm{m}^{-3}$ hydrophilic polymer treatment. Higher overwintering rate was associated with lower coir dust and hydrophilic polymer content. The low temperature injures the growth of plant roots, and cold weather freezes the surface soil sometimes, inflicting damage on shallow roots such as those planted in green roofs. Another type of winter root injury is caused by frost heaving, which is the result of the formation of ice lenses in the soil caused by a segregation of the soil water. Ice lenses are growing from below and pushed upward, which cause the plants to move upwards in the soil, sometimes pushing them out of the soil altogether. This can break many of the fine roots (Cleavitt et al., 2008). Injury or death usually follows if roots are broken or the shoots and exposed roots become dried out. Frost heaving is most likely to occur in fine-textured soils with high water content (Tarnocai and Bockheim, 2017). The higher coir dust and hydrophilic polymer content resulted in higher water content in substrates, thus leading to the low rate of overwintering. 
The result also confirmed that the self-sown seedling number was affected by the composition of substrate, hydrophilic polymer rate. The germination of seed associated with moisture content, suitable temperature, and adequate supply of oxygen (FinchSavage and Leubner-Metzger, 2006). However, long-term high moisture maintenance may result in seeds of some species rotting. As to Agastache rugosa, there was more number of seed starting seedlings under the $\mathrm{C}_{1} \mathrm{P}_{4}$ treatment by the perlite based (perlite) substrate, compared to coir dust based substrate.

\section{Conclusions}

Our results indicated that higher moisture was retained in substrates with hydrophilic polymers compared to that in the control substrate. We found that Agastache rugosa had a high drought tolerance capable of withstanding extended dry conditions in green roofs. The $\mathrm{C}_{4} \mathrm{P}_{1}$ substrate, which has high coir dust content, is not recommended for Agastache rugosa because of its low growth and poor ornamental quality, especially in the rainy season. Addition of $1.0 \mathrm{~kg} \cdot \mathrm{m}^{-3}$ hydrophilic polymer to substrate $\mathrm{C}_{1} \mathrm{P}_{4}$ increased the inflorescences number and resulted in good growth and ornamental quality both under drought and rainy seasons. Higher overwintering rate was associated with lower coir dust and hydrophilic polymer content. A greater number of seeds started seedling in substrate $\mathrm{C}_{1} \mathrm{P}_{4}$, especially at rates of 1.0 and $2.0 \mathrm{~kg} \cdot \mathrm{m}^{-3}$. These results indicated that the addition of $1.0 \mathrm{~kg} \cdot \mathrm{m}^{-3}$ hydrophilic polymer to substrate $\mathrm{C}_{1} \mathrm{P}_{4}$ is optimal for Agastache rugosa in green roofs. Further research studies are needed to determine the physical and chemical properties of substrates with different amounts of hydrophilic polymers. In addition, a follow up study on the effects of hydrophilic polymers on overwintering and the growth of Agastache rugosa in the second year would be useful.

Acknowledgements. This research was supported by Basic Science Research Program through the National Research Foundation of Korea (NRF) funded by the Ministry of Science, ICT \& Future Planning (2015RIC1A2A01052633).

\section{REFERENCES}

[1] Álvarez, S., Bañón, S., Sánchez-Blanco, M. J. (2013): Regulated deficit irrigation in different phenological stages of potted geranium plants: water consumption, water relations and ornamental quality. - Acta Physiologiae Plantarum 35: 1257-1267.

[2] Andry, H., Yamamoto, T., Irie, T., Moritani, S., Inoue, M., Fujiyama, H. (2009): Water retention, hydraulic conductivity of hydrophilic polymers in sandy soil as affected by temperature and water quality. - Journal of Hydrology 373: 177-183.

[3] Bai, W., Zhang, H., Liu, B., Wu, Y., Song, J. (2010): Effects of super-absorbent polymers on the physical and chemical properties of soil following different wetting and drying cycles. - Soil Use and Management 26: 253-260.

[4] Baran, A., Zaleski, T., Kulikowski, E., Wieczorek, J. (2015): Hydrophysical and biological properties of sandy substrata enriched with hydrogel. - Polish Journal of Environmental Studies 24: 2355-2362.

[5] Bates, A. J., Sadler, J. P., Greswell, R. B., Mackay, R. (2015): Effects of recycled aggregate growth substrate on green roof vegetation development: A six year experiment. - Landscape and Urban Planning 135: 22-31. 
[6] Beattie, D. J., Berghage, R. (2004): Green roof media characteristics: the basics. - Proc. of 2nd North American Green Roof Conference: Greening Rooftops for Sustainable Communities, Portland, Oregon, pp. 411-416.

[7] Butler, C., Butler, E., Orians, M. (2012): Native plant enthusiasm reaches new heights: Perceptions, evidence, and the future of green roofs. - Urban Forestry \& Urban Greening 11: $1-1$.

[8] Caser, M., Lovisolo, C., Scariot, V. (2017): The influence of water stress on growth, ecophysiology and ornamental quality of potted Primula vulgaris 'Heidy' plants. New insights to increase water use efficiency in plant production. - Plant Growth Regulation 83: 361-373.

[9] Cho, M. S., Park, Y. Y., Jun, H. J., Chung, J. B. (2006): Growth of Gerbera in mixtures of coir dust and perlite. - Horticulture Environment and Biotechnology 47: 211-216.

[10] Choi, S., Seo, Y. (2007): Effect of sta-green and activated carbon on growth of Agastache rugosa in green house. - Korean Journal of Plant Resources 20: 255-257.

[11] Cleavitt, N. L., Fahey, T. J., Groffman, P. M., Hardy, J. P., Henry, K. S., Driscoll, C. T. (2008): Effects of soil freezing on fine roots in a northern hardwood forest. - Canadian Journal of Forest Research 38: 82-91.

[12] Dorraji, S. S., Golchin, A., Ahmadi, S. (2010): The effects of hydrophilic polymer and soil salinity on corn growth in sandy and loamy soils. - Clean-Soil, Air, Water 38: 584591.

[13] Dunnett, N., Kingsbury, N. (2004): Planting Green Roofs and Living Walls. - Timber Press, Portland, Oregon.

[14] Dunnett, N., Nolan, A. (2004): Effect of substrate depth and supplementary watering on the growth of nine herbaceous perennials in a semi-extensive green roof. - Acta Horticulturae 643: 305-309.

[15] Farrell, C., Ang, X. Q., Rayner, J. P. (2013): Water-retention additives increase plant available water in green roof substrates. - Ecological Engineering 52: 112-118.

[16] Finch Savage, W. E., Leubner Metzger, G. (2006): Seed dormancy and the control of germination. - New Phytologist 171: 501-523.

[17] Geesing, D., Schmidhalter, U. (2004): Influence of sodium polyacrylate on the waterholding capacity of three different soils and effects on growth of wheat. - Soil Use and Management 20: 207-209.

[18] Hammond, H. E., Norcini, J. G., Wilson, S. B., Schoellhorn, R. K., Miller, D. L. (2007): Growth, flowering, and survival of firewheel Gaillardia pulchella Foug. based on seed source and growing location. - Native Plants Journal 8: 25-39.

[19] Han, Y., Yu, X., Yang, P., Li, B., Xu, L., Wang, C. (2013): Dynamic study on water diffusivity of soil with super-absorbent polymer application. - Environmental Earth Sciences 69: 289-296.

[20] Jobin, P., Carno, J., Bernier, P. Y., Dansereau, B. (2004): Impact of two hydrophilic acrylic-based polymers on the physical properties of three substrates and the growth of Petunia xhybrida 'Brilliant Pink'. - Journal of the American Society for Horticultural Science 129: 449-457.

[21] Koshita, Y., Takahara. T. (2004): Effect of water stress on flower-bud formation and plant hormone content of satsuma mandarin (Citrus unshiu Marc.). - Scientia Horticulturae 99: 301-307.

[22] Lovelli, S., Perniola, M., Ferrara, A., Di Tommaso, T. (2007): Yield response factor to water (Ky) and water use efficiency of Carthamus tinctorius L. and Solanum melongena L. - Agricultural Water Management 92: 73-80.

[23] Nagase, A., Dunnett, N. (2010): Drought tolerance in different vegetation types for extensive green roofs: effects of watering and diversity. - Landscape and Urban Planning 97: 318-327. 
[24] Nagase, A., Dunnett, N. (2011): The relationship between percentage of organic matter in substrate and plant growth in extensive green roofs. - Landscape and Urban Planning 103: 230-236.

[25] Nardini, A., Andri, S., Crasso, M. (2012): Influence of substrate depth and vegetation type on temperature and water runoff mitigation by extensive green roofs: shrubs versus herbaceous plants. - Urban Ecosystems 15: 697-708.

[26] Netto, A. T., Campostrini, E., de Oliveira, J. G., Bressan-Smith, R. E. (2005): Photosynthetic pigments, nitrogen, chlorophyll a fluorescence and SPAD-502 readings in coffee leaves. - Scientia Horticulturae 104: 199-209.

[27] Olszewski, M. W., Holmes, M. H., Young, C. A. (2010): Assessment of physical properties and stonecrop growth in green roof substrates amended with compost and hydrogel. - HortTechnology 20: 438-444.

[28] Oschmann, C., Kobayashi, N., Perkuhn, C., Grüneberg, H., Wissemeier, A. H. (2007): Study to expand the range of wild plants for extensive roof greening systems using superabsorbent polymers (SAP). - Acta Horticulturae 813: 421-426.

[29] Papafotiou, M., Pergialioti, N., Tassoula, L., Massas, I., Kargas, G. (2013): Growth of native aromatic xerophytes in an extensive Mediterranean green roof as affected by substrate type and depth and irrigation frequency. - HortScience 48: 1327-1333.

[30] Passioura, J. (2006): Increasing crop productivity when water is scarce-from breeding to field management. - Agricultural Water Management 80: 176-196.

[31] Razmjoo, K., Heydarizadeh, P., Sabzalian, M. R. (2008): Effect of salinity and drought stresses on growth parameters and essential oil content of Matricaria chamomile. International Journal of Agriculture and Biology 10: 451-454.

[32] Rowe, D. B., Monterusso, M. A., Rugh, C. L. (2006): Assessment of heat-expanded slate and fertility requirements in green roof substrates. - HortTechnology 16: 471-477.

[33] Santamouris, M. (2014): Cooling the cities-a review of reflective and green roof mitigation technologies to fight heat island and improve comfort in urban environments. - Solar Energy 103: 682-703.

[34] Savi, T., Marin, M., Boldrin, D., Incerti, G., Andri, S., Nardini, A. (2014): Green roofs for a drier world: Effects of hydrogel amendment on substrate and plant water status. Science of the Total Environment 490: 467-476.

[35] Schafer, P. (2011): The Chinese Medicinal Herb Farm: A Cultivator's Guide to SmallScale Organic Herb Production. - Chelsea Green Publishing, White River Junction, Vermont.

[36] Tarnocai, C., Bockheim, J. G. (2017): Soils of Cold and Permafrost Affected Landscapes. - In: Richardson, D. et al. (eds.) The International Encyclopedia of Geography. Wiley, New York.

[37] Wang, S. Y., Camp, M. J. (2000): Temperatures after bloom affect plant growth and fruit quality of strawberry. - Scientia Horticulturae 85: 183-199.

[38] Xu, H., Yoon, Y. H., Choi, E. Y., Kang, H. K., Ju, J. H. (2014): Combination effects of the use of hydrophilic polymer for green roof substrate and rainy season condition on growth and biomass partitioning of Aster koraiensis. - Journal of Food Agriculture and Environment 12: 1177-1181.

[39] Yamani, H., Mantri, N., Morrison, P. D., Pang, E. (2014): Analysis of the volatile organic compounds from leaves, flower spikes, and nectar of Australian grown Agastache rugosa. - BMC complementary and Alternative Medicine 14: 495.

[40] Young, T. M., Cameron, D. D., Phoenix, G. K. (2017): Increasing green roof plant drought tolerance through substrate modification and the use of water retention gels. Urban Water Journal 14: 551-560. 